\title{
On the Mobility and Fault Tolerance of Closed Chain Manipulators with Passive Joints
}

\author{
Pål Johan From ${ }^{1}$ Jan Tommy Gravdahl ${ }^{1}$
} ${ }^{1}$ Department of Engineering Cybernetics, Norwegian University of Science and Technology, N-7491 Trondheim,
Norway. E-mail: \{from, tommy.gravdahl\}@itk.ntnu.no

\begin{abstract}
A systematic analysis of the mobility of closed chain manipulators with passive joints is presented. The main observation in this paper is that the mobility of the manipulator, considering the passive joints only, should always be zero. Further, for the manipulator to be fault tolerant, the mobility should remain zero when actuator failure occurs for an arbitrary joint. We present a simple and rigorous approach to the problem of finding the smallest set of active joints for which the manipulator remains equilibrated with respect to free swinging joint failure in any joint. Several examples of how to choose the active joints for different mechanisms to guarantee that the manipulator is equilibrated and fault tolerant are presented.
\end{abstract}

Keywords: Robotics, kinematics, mobility, fault tolerance.

\section{Introduction}

Closed chain manipulators such as parallel manipulators and cooperating serial manipulators have many advantages over their serial counterparts. Parallel manipulators are stiffer, faster and more accurate than serial manipulators at the cost of a smaller workspace. Cooperating robots can handle heavier and larger objects than serial manipulators and are thus the preferred choice in many applications. Both parallel and cooperating robots are widely used especially in remote and harsh environments where humans can not or do not want to operate. The need for a rigorous theory on what happens when joint failure occurs is thus important to be able to cope with unforeseen events such as joint failures.

This paper discusses the effect that passive joints have on the mobility of parallel manipulators. The main motivation and also the main example used throughout the paper is joint failure. We study the ability of the mechanism to remain equilibrated when free-swinging joint failure (FSJF) occurs, see Tinós et al. (2006). FSJF is also referred to as torque failure in the literature (Matone and Roth, 1999).

We will denote a manipulator equilibrated if it can resist a wrench in an arbitrary direction, either through kinematic constraints or through actuator torques. We obtain this if the manipulator, considering the passive joints only, has mobility equal to zero, i.e. we do not want the passive joints to allow any motion when the active joints are locked. If this property is satisfied this is the same as guaranteeing that manipulator does not have an unstable singularity, following the classification in Matone and Roth (1999).

For non-overconstrained mechanisms, i.e. when there are no redundant constraints, we can apply the well known Grübler formula. The active joints can be chosen arbitrarily as long as the manipulator remains non-overconstrained and the self-motion is considered.

For overconstrained mechanisms, there are many approaches to determine the mobility. In Dai et al. (2006) the mobility of the mechanism is found from the constraint space. The constraints of the system are found systematically and the redundant constraints are identified. The mobility is then found by adding the degrees of freedom represented by these redundant con- 
straints to the Grübler formula for non-overconstrained mechanisms. This approach illustrates well the effect of redundant constraints in the mechanism.

The mobility can also be found by the motion space as in Rico et al. (2003) and Rico et al. (2006). The degree of freedom of the motion of the end effector is first found. Then the degree of freedom of the self-motion manifold of each chain is added. By this approach the redundant constraints are not found directly. This approach also gives valuable in-sight on where to place redundant actuators in the mechanism.

We present a systematic and rigorous analysis of the mobility of closed chain mechanisms based on the theory of twists. The analysis makes it possible to calculate the mobility of the mechanism based on the number and type of joints in each sub-chain. We then determine the minimum set of active joints needed for the manipulator to be equilibrated and fault tolerant. The mechanism needs to be equilibrated not only with respect to forces acting on the end effector, but also with respect to forces acting on the chains. Thus, in addition to the end-effector motion we also need to consider the internal motion of each chain to guarantee that the mechanism is equilibrated.

We present several examples of how to apply the theory presented to different mechanisms. For three types of mechanisms, exceptional linkage and trivial linkage of type I and II, we show how to choose the minimum number of active joints so that the mechanisms are equilibrated and fault tolerant.

\section{Rigid Body Motion}

This section gives the background of mathematical modelling of rigid body motion. For a detailed overview of the topic, the reader is referred to Murray et al. (1994) and Meng et al. (2007).

We will use the special Euclidean group $S E(3)$ to represent the configuration space of a rigid body. In addition to its group structure, $S E(3)$ is a differentiable manifold, and is what is known as a Lie group. $S E(3)$ is thus a matrix Lie group and can be written by homogeneous coordinates

$$
S E(3)=\left\{\left[\begin{array}{cc}
R & p \\
0 & 1
\end{array}\right] \mid p \in \mathbb{R}^{3}, R \in S O(3)\right\}
$$

where $S O(3)$ is the 3 -dimensional special orthogonal group. An element $g \in S E(3)$ represents a rotation and a displacement of a rigid body relative to a reference configuration. The manifold structure of $S E(3)$ is given by

$$
\Phi: S O(3) \ltimes \mathbb{R}^{3} \rightarrow S E(3):(R, p) \mapsto\left[\begin{array}{cc}
R & p \\
0 & 1
\end{array}\right] .
$$

Associated with every Lie group $G$ is its Lie algebra $\mathfrak{g}$ which is defined as the tangent space of $G$ at the identity $e$ and is written as $\mathfrak{g} \triangleq T_{e} G$. A vector space $V$ is a Lie algebra if there exists a bilinear operation given by the matrix commutator $\left[v_{1}, v_{2}\right]=v_{1} v_{2}-v_{2} v_{1}$. The Lie algebra $s e(3)$ of $S E(3)$ consist of all $4 \times 4$ matrices

$$
s e(3)=\left[\begin{array}{ll}
\hat{\omega} & v \\
0 & 0
\end{array}\right]
$$

where $v \in \mathbb{R}^{3}$ and $\hat{\omega}$ is the skew-symmetric matrix representation of $\omega \in \mathbb{R}^{3}$ given by

$$
\hat{\omega}=\left[\begin{array}{ccc}
0 & -\omega_{3} & \omega_{2} \\
\omega_{3} & 0 & -\omega_{1} \\
-\omega_{2} & \omega_{1} & 0
\end{array}\right] \in s o(3) .
$$

An element of se(3) can be represented by the twist coordinates $\xi=\left[\begin{array}{ll}v^{\top} & \omega^{\top}\end{array}\right]^{\top} \in \mathbb{R}^{6}$ which can be identified with the twist $\hat{\xi} \in \operatorname{se}(3)$ by the map

$$
\wedge: \mathbb{R}^{6} \rightarrow \operatorname{se}(3): \xi=\left[\begin{array}{c}
v \\
\omega
\end{array}\right] \mapsto \hat{\xi}=\left[\begin{array}{cc}
\hat{\omega} & v \\
0 & 0
\end{array}\right] \in \operatorname{se}(3) .
$$

The exponential map

$$
\exp : \operatorname{se}(3) \rightarrow S E(3): \hat{\xi} \mapsto e^{\hat{\xi}}
$$

defines a local diffeomorphism taking the zero vector of $s e(3)$ to the identity element of $S E(3)$. Physically $e^{\theta \hat{\xi}}$, $\theta \in \mathbb{R}$ corresponds to a screw motion along the axis of a fixed $\xi$. Denote by $L_{g}$ and $R_{g}$ the left and right translation map, respectively. The differential $L_{g *}$ of $L_{g}$ defines the body velocity and the differential $R_{g *}$ of $R_{g}$ defines spatial ${ }^{1}$ velocity of a rigid body. Then for a trajectory $g(t) \in S E(3), t \in(-\epsilon, \epsilon)$, the body velocity of the rigid body is given by

$$
\hat{V}^{b}=L_{g(t)^{-1} *} \cdot \dot{g}(t)=\left[\begin{array}{cc}
R^{\top} \dot{R} & R^{\top} \dot{p} \\
0 & 0
\end{array}\right]=\left[\begin{array}{cc}
\hat{\omega} & v \\
0 & 0
\end{array}\right]
$$

while the spatial velocity is given by $\hat{V}^{s}=R_{g^{-1} *}$. $\dot{g}$. The body and spatial velocities are related by the Adjoint map

$$
V^{s}=\operatorname{Ad}_{g} V^{b}
$$

where $g=(R, p)$ and

$$
\operatorname{Ad}_{g}=\left[\begin{array}{cc}
R & \hat{p} R \\
0 & R
\end{array}\right]
$$

We will write the twist of joint $i$ as $\mathcal{G}_{i}$ and the twist system of chain $j$ as

$\overline{\mathcal{M}}_{j}=\left(\mathcal{G}_{1}, \mathcal{G}_{2}, \ldots, \mathcal{G}_{n}\right)=\left(\mathcal{M}_{j 1}, \mathcal{M}_{j 2}, \ldots, \mathcal{M}_{j n}\right)$.

\footnotetext{
${ }^{1}$ In this context, spatial means that the velocity is given with respect to a globally defined coordinate system. We will also use spatial for the 3-dimensional space, as opposed to the planar case.
} 
where we use the second notation $\mathcal{M}_{j i}$ when we need to clarify what chain the joint belongs to. We use the same notation for the joint angles, i.e. $\theta_{j i}$. The twist system describes the motion of the end effector for the open chain.

We will introduce the following notation from Dai et al. (2006) to represent sets of twists or wrenches. Braces $\{\cdot\}$ are used to indicate a set that contains unique elements while angle brackets $\langle\cdot\rangle$ are used to indicate multisets which may contain multiple entries of each element. We will use cardinality (card) to give the number of elements in $\left\langle\overline{\mathcal{M}}_{j}\right\rangle$ or $\left\{\overline{\mathcal{M}}_{j}\right\}$. For $\left\{\overline{\mathcal{M}}_{j}\right\}$, the cardinality is equal to the dimension. Let the parallel manipulator

$$
\mathcal{M}=\mathcal{M}_{1}\left\|\mathcal{M}_{2}\right\| \cdots \| \mathcal{M}_{k}
$$

consist of $k$ serial manipulator sub-chains that share a common base and a common end effector. The set of end-effector motions is defined as (Meng et al., 2007)

$$
C_{\mathcal{M}}=C_{\mathcal{M}_{1}} \cap C_{\mathcal{M}_{2}} \cap \cdots \cap C_{\mathcal{M}_{k}},
$$

where $C_{\mathcal{M}_{j}}$ is the set of rigid transformations that $\mathcal{M}_{j}$ generates without loop constraints. $C_{\mathcal{M}}$ defines the configurations of the end effector with the loop constraints imposed.

We are interested in the passive motion, i.e. the motion due to the passive joints when the active joints are fixed. We denote this by

$$
\mathcal{M}_{P}=\mathcal{M}_{P 1}\left\|\mathcal{M}_{P 2}\right\| \cdots \| \mathcal{M}_{P k}
$$

where $\mathcal{M}_{P j}$ consists of only the passive joints of manipulator $j$.

Although only the passive joints are considered, the twists of the passive joints depend on the configuration of the active joints. The twist of joint $i$ is given by

$$
\mathcal{G}_{i}^{\prime}=\operatorname{Ad}_{g_{(i-1)}} \mathcal{G}_{i}
$$

where $g_{i} \in S E(3)$ is the transformation from the base to joint $i$. We will assume it implicitly understood that the twists, as written in (10), are transformed according to (14), and thus write $\mathcal{G}$ for $\mathcal{G}^{\prime}$.

We will find the mobility $D$ considering the passive joints only. If the mobility of the mechanism is zero we can conclude that the mechanism is equilibrated with respect to any external force. On the other hand, if $D>0$ an additional condition needs to be satisfied for the mechanism to be equilibrated. This is not considered in this paper.

We will denote a mechanism equilibrated if the following is satisfied:

Definition 2.1. A manipulator $\mathcal{M}$ is denoted equilibrated with respect to an external wrench $F_{\text {ext }}=$ $\left[\begin{array}{ll}f^{\top} & \tau^{\top}\end{array}\right]^{\top}$ where $f, \tau \in \mathbb{R}^{3}$, if $\mathcal{M}$, either through kinematic constraints or through actuator torque, can produce a wrench opposite to $F_{\text {ext }}$, i.e. $\mathcal{M}$ can produce the wrench $-k F_{\text {ext }}$ for some $k>0$. In the case that an arbitrary wrench that can be accommodated by the kinematic constraints, we will say that the manipulator is passively sustained. When an arbitrary wrench can be produced by the actuation torque, we will denote it actively equilibrated.

Note that we do not require that the manipulator can resist any external wrench, only that it can produce a wrench of a given type and direction.

In the next section we start by looking at the case when the constraints are linearly independent, i.e. nonoverconstrained mechanisms. This is very restrictive, and the results may be misleading if they are not used with caution. Non-overconstrained mechanisms, are however, easy to understand and many of the results from the non-overconstrained case are also true for overconstrained mechanisms.

\section{Non-overconstrained Mechanisms}

We start by looking at the mobility of nonoverconstrained mechanisms when passive joints are present and find a set of rules to guarantee that the manipulator is fault tolerant.

\subsection{The Planar Case}

For the planar case the mobility of the closed chain mechanism is given by Grübler's formula

$$
D=3 N-\sum_{i=1}^{n}\left(3-f_{i}\right) .
$$

where $N$ is the number of links in the mechanism and $n$ is the number of joints. $f_{i}$ is the degree of freedom of joint $i$ which is 1 for the lower pairs. Grübler's formula then becomes

$$
D=3 N-2 n .
$$

We will start by a simple result which states that a nonoverconstrained closed chain manipulator is always resistant to external forces if the number of active joints is equal or larger than the dimension of the end-effector motion $Q$. The only requirement is that the manipulator remains non-overconstrained when the joint chosen as active is considered fixed. This is the same as requiring that every component of the end-effector motion and the internal motion of each chain is generated by at least one joint, or to require that only joints that are not locked are chosen. All the results and examples 
can easily be generalised to 2 or 3 degree of freedom joints. For simplicity we restrict ourselves to the case $f_{i}=1$.

Proposition 3.1. Given a desired end-effector motion type $Q$ with $m=\operatorname{dim}(Q)$ and a planar parallel manipulator $\mathcal{M}=\mathcal{M}_{1}\|\cdots\| \mathcal{M}_{k}$. Then if $m$ joints, which are not already locked, are chosen active, then $\mathcal{M}_{P}$ is locked and $\mathcal{M}$ is equilibrated.

Proof. Assume all joints passive so that $D=m$. Then by choosing the active joints we need to reduce the degree of freedom of the remaining joints $\left(\mathcal{M}_{P}\right)$ to zero. We have that for each joint that is chosen active, $n$ in Equation (16) is reduced by one. Disregarding this joint, i.e. assuming it fixed, the number of links $N$ is also reduced by one. The degree of freedom of the remaining passive joints when the mechanism remains non-overconstrained is thus given by

$$
\begin{aligned}
D_{1} & =3(N-1)-2(n-1) \\
& =3 N-2 n-3+2 \\
& =D-1 .
\end{aligned}
$$

Repeating this $m$ times, we will have $D_{m}=D-m$ where the subscript in $D_{i}$ is the number of active joints in the mechanism. The mobility of $\mathcal{M}_{P}$ is thus zero.

This is consistent with the result that at least $m$ actuators are needed to generate a motion of dimension $m$. Hence, by choosing $m$ joints active, the mechanism $\mathcal{M}_{A}$, considering both active and passive joints, generates $Q$ and $\mathcal{M}_{P}$ is equilibrated with respect to any external disturbance.

\subsection{The Spatial Case}

For the three-dimensional Euclidean space, Grübler's formula becomes

$$
D=6 N-\sum_{i=1}^{n}\left(6-f_{i}\right),
$$

where $f_{i}$ is 1 for the 1 -dimensional lower pairs and we write

$$
D=6 N-5 n .
$$

Proposition 3.2. Given a desired end-effector motion type $Q$ with $m=\operatorname{dim}(Q)$ and a spatial parallel manipulator $\mathcal{M}=\mathcal{M}_{1}\|\cdots\| \mathcal{M}_{k}$. Then if $m$ joints, which are not already locked, are chosen active, then $\mathcal{M}_{P}$ is locked and $\mathcal{M}$ is equilibrated.
Proof. The proof follows from the planar case. By choosing the active joints both $n$ and $N$ are reduced by one. The degree of freedom of the remaining passive joints when the mechanism remains nonoverconstrained is thus given by

$$
\begin{aligned}
D_{1} & =6(N-1)-5(n-1) \\
& =6 N-5 n-6+5 \\
& =D-1 .
\end{aligned}
$$

Repeating this $m$ times, we will have that the mobility of $\mathcal{M}_{P}$ is zero.

Thus we see that we can derive three equivalent rules on how to choose the active joints for nonoverconstrained mechanisms:

- Choose the active joint from the set of joints that is not locked due to the kinematic constraints, considering the previously chosen active joints as fixed.

- Choose the active joint such that the mechanism remains non-overconstrained.

- Choose the active joints such that every component of the end-effector motion and the internal motion of each chain is generated by at least one active joint.

From Propositions 3.1 and 3.2 we see that the active joints cannot be placed arbitrarily. We need to place at least one active joint for each degree of freedom of the self-motion manifold in the respective chain. Denote the freedom of chain $j$ by $D^{j}$. This is found by applying Grübler's formula to each chain. For the manipulator to be equilibrated we need to place $D^{j}$ active joints in the set of joints that generate the self-motion.

Once this is done, we need to place the remaining $\operatorname{dim}(Q)$ active joints. Each degree of freedom of $Q$ corresponds to a one degree of freedom motion. Then, for each component of the motion $Q$, one active joint must be chosen among the joints that generate the corresponding 1 DOF motion. This will guarantee that every "direction" of the end-effector motion is actively equilibrated when the active joints are locked.

\subsection{Fault Tolerance}

For the manipulator to be fault tolerant we need to place the redundant actuators in a similar manner. Redundant actuators are the actuators that will guarantee that the manipulator remains actively equilibrated when actuator failure occurs.

For the chain to be fault tolerant we need $D^{j}+1$ active joints in $\mathcal{M}_{j}$ for all chains that are not passively sustained, i.e. whenever $D^{j}>0$. These active 
joints must be chosen among the joints that generate the self-motion. This guarantees that the chains are fault tolerant. In addition, for each subgroup of $Q$, one redundant active joint must be chosen among the joints that generate the motion of the respective subgroup. Note that one joint can generate a motion in more than one sub-group. We also note that if a redundant joint is implemented in a chain, this will also make the mechanism fault tolerant for joint failures in any active joint that generate a motion in the same subgroup as the redundant joint.

The results presented so far only apply for nonoverconstrained manipulators. The results do, however, with a few modifications, give valuable in-sight into the mobility of overconstrained manipulators. A spatial manipulator may generate motion that is not identified by applying the spatial Grübler formula. If a subset of the joints generate motion in the plane, the planar Grübler formula must be applied to these joints to find the dimension of the motion generated by these joints. In fact, this is the case for all the subgroups. To guarantee that the passive joints do not generate any motion, the Grübler formula must be applied to all the ten subgroup of $S E(3)$, with $d$ being the dimension of the subgroup

$$
D=d N-\sum_{i=1}^{n}\left(d-f_{i}\right) .
$$

Here, only the joints or sets of joints that generate motion in the given subgroup are considered.

Thus, for the spatial case, the mobility needs to be checked for all the ten subgroups of $S E(3)$ in addition to the spatial Grübler formula. Similarly for the planar case the mobility needs to be checked for all the subgroups of $S E(2)$, i.e. $T(1), T(2)$ and $S O(2)$, in addition to the planar Grübler formula.

The same is true for the self-motion. A chain in a spatial mechanism can have self-motion even if the mobility by the spatial Grübler formula is zero. Also in this case, the mobility needs to be checked for all the subgroups of $S E(3)$. When the mobility is found to be greater than zero, the active and the redundant joints can be chosen arbitrarily among the joints that generate the respective motion.

We see that the Grübler formula is not very well suited for overconstrained mechanisms. In the following we thus show how to use the theory of twists to find the mobility of general mechanisms by applying the Grübler formula only once or without applying the Grübler formula at all.

\section{Overconstrained Mechanisms}

When the constraints are not linearly independent, the mechanism is over-constrained, i.e. some of the constraints are redundant and have no effect on the mobility. Based on the approach in Dai et al. (2006) we first identify the constraints that are common for all chains and eliminate the redundancy in this set. This set is easily identified as the intersection of the constraint space of all the chains. Further the constraints that constrain each chain to the end effector-motion, the end effector constraint system, are identified and again the redundant constraints are found from this set. The approach presented in Dai et al. (2006) is based on the screw system of the mechanism and represents the constraint space as reciprocal screws. Here, we apply the same general idea as in Dai et al. (2006). The approach is based on an analysis of the sub-algebras and sub-manifolds of the Lie Algebra se(3) and their cotangent spaces.

The approach is general in the sense that no classification of the mechanism is required. As pointed out in Rico et al. (2003), the classification of the mechanism is not needed to determine its mobility. However, in our setting, the classification is important in the sense that it tells us where to place the active joints. As for the non-overconstrained case, the active joints cannot be placed arbitrarily in the mechanism. We will see that only in very special cases can the active joints be arbitrarily chosen in the mechanism.

The approach in Dai et al. (2006) is based on the constraint space formulation. It is also shown that the mobility can be found by the motion space as in Rico et al. (2003) and Rico et al. (2006). By this approach the chains are also classified and it is straight forward to determine the effect a passive joint has on the mechanism. We will use the motion space approach to set up a set of simple rules on where to place the active joints in the mechanism in order for the mechanism to be equilibrated. This set of rules naturally leads to an approach on how to choose actuator redundancy most efficiently to make the manipulator resistant to joint failures.

\subsection{The Constraint Space}

To find the mobility from the constraint space as in Dai et al. (2006), we start by denoting the motion space of the chain $j$ as

$$
\overline{\mathcal{M}}_{j}=\left(\mathcal{G}_{1}, \mathcal{G}_{2}, \ldots, \mathcal{G}_{n_{j}}\right)
$$

where $\mathcal{G}_{i}$ is the twist of joint $i$ and $n_{j}$ is the number of joints in chain $j$. Recall that braces $\{\cdot\}$ are used to indicate a set that contains unique elements while 
angle brackets $\langle\cdot\rangle$ are used to indicate multisets which may contain multiple entries of each element.

We will denote the constraint system of chain $j$ as

$$
\overline{\mathcal{M}}_{j}^{C}=\overline{\mathcal{M}}_{j}^{\perp}
$$

where $\overline{\mathcal{M}}_{j}^{\perp}=\left\{F \in \mathbb{R}^{6} \mid F \cdot V=0, \forall V \in \overline{\mathcal{M}}_{j}\right\}$ which is the vanishing of the reciprocal product of Ball (Lipkin and Duffy, 2002). This represents the constraints imposed on the end effector by chain $j$. Note that we cannot identify the self-motion from $\overline{\mathcal{M}}_{j}^{C}$.

Further we will define mechanism motion as the union of all the twists in the system

$$
\overline{\mathcal{M}}_{M}=\overline{\mathcal{M}}_{1} \cup \overline{\mathcal{M}}_{2} \cup \cdots \cup \overline{\mathcal{M}}_{k},
$$

evaluated at $g \in C_{\mathcal{M}_{1}} \cap \cdots \cap C_{\mathcal{M}_{k}}$. The end-effector constraints is given as the union of the constraints of each chain,

$$
\overline{\mathcal{M}}_{E}^{C}=\overline{\mathcal{M}}_{1}^{C} \cup \overline{\mathcal{M}}_{2}^{C} \cup \cdots \cup \overline{\mathcal{M}}_{k}^{C},
$$

evaluated at $g \in C_{\mathcal{M}_{1}} \cap \cdots \cap C_{\mathcal{M}_{k}}$. From this we can find the constrained motion of the end effector

$$
\overline{\mathcal{M}}_{E}=\left(\overline{\mathcal{M}}_{E}^{C}\right)^{\perp} .
$$

The intersection of all the constraints are further given by

$$
\overline{\mathcal{M}}_{M}^{C}=\overline{\mathcal{M}}_{1}^{C} \cap \overline{\mathcal{M}}_{2}^{C} \cap \cdots \cap \overline{\mathcal{M}}_{k}^{C},
$$

or alternatively

$$
\overline{\mathcal{M}}_{M}^{C}=\left(\overline{\mathcal{M}}_{M}\right)^{\perp} .
$$

With the notation of braces and angle brackets, each of the subsets introduced in this section is given by

$$
\begin{aligned}
\left\{\overline{\mathcal{M}}_{M}\right\} & =\bigcup_{j=1}^{k} \overline{\mathcal{M}}_{j}, & \left\langle\overline{\mathcal{M}}_{M}\right\rangle & =\sum_{j=1}^{k} \overline{\mathcal{M}}_{j}, \\
\left\{\overline{\mathcal{M}}_{E}^{C}\right\} & =\bigcup_{j=1}^{k} \overline{\mathcal{M}}_{j}^{\perp}, & \left\langle\overline{\mathcal{M}}_{E}^{C}\right\rangle & =\sum_{j=1}^{k} \overline{\mathcal{M}}_{j}^{\perp}, \\
\left\{\overline{\mathcal{M}}_{E}\right\} & =\bigcap_{j=1}^{k} \overline{\mathcal{M}}_{j}, & \left\{\overline{\mathcal{M}}_{M}^{C}\right\} & =\sum_{j=1}^{k} \overline{\mathcal{M}}_{j}^{C}, \\
\left\{\overline{\mathcal{M}}_{E}\right\} & =\left\{\overline{\mathcal{M}}_{E}^{C}\right\}^{\perp} & \left\{\overline{\mathcal{M}}_{M}^{C}\right\} & =\left\{\overline{\mathcal{M}}_{M}\right\}^{\perp} \\
& =\left\langle\overline{\mathcal{M}}_{E}^{C}\right\rangle^{\perp}, & & =\left\langle\overline{\mathcal{M}}_{M}\right\rangle^{\perp} .
\end{aligned}
$$

Thus, the collection of all constraints is given in $\left\langle\overline{\mathcal{M}}_{E}^{C}\right\rangle$, including repeated elements. The first step is to factorise out all the constraints that are common for all chains. The "directions" of the end effector represented by these constraints can be considered the most robust directions as they are constrained by all the sub-chains in the mechanism. The constraints that are common for all sub-chains is given by $\left\{\overline{\mathcal{M}}_{M}^{C}\right\}$. We will say that a single subchain cannot have redundant constraints (as seen from the other chains or the end effector). This is always true. Because $\left\{\overline{\mathcal{M}}_{M}^{C}\right\}$ is the same for all chains we can write

$$
\left\langle\overline{\mathcal{M}}_{M}^{C}\right\rangle=k \cdot\left\{\overline{\mathcal{M}}_{M}^{C}\right\} .
$$

We see that $\left\langle\overline{\mathcal{M}}_{M}^{C}\right\rangle$ is $(k-1)$ times redundant.

For each chain we can factorise out this part by taking

$$
\left\{\overline{\mathcal{M}}_{j}^{C}\right\}=\left\{\overline{\mathcal{M}}_{M}^{C}\right\} \cup\left\{\overline{\mathcal{M}}_{C j}^{C}\right\}
$$

where $\left\{\overline{\mathcal{M}}_{M}^{C}\right\} \cap\left\{\overline{\mathcal{M}}_{C j}^{C}\right\}=\emptyset$. We can add the multisets of Equation (30) and get

$$
\begin{aligned}
\left\langle\overline{\mathcal{M}}_{E}^{C}\right\rangle & =\left\langle\overline{\mathcal{M}}_{M}^{C}\right\rangle+\left\langle\overline{\mathcal{M}}_{C}^{C}\right\rangle \\
& =k \cdot\left\{\overline{\mathcal{M}}_{M}^{C}\right\}+\left\langle\overline{\mathcal{M}}_{C}^{C}\right\rangle
\end{aligned}
$$

where $\left\langle\overline{\mathcal{M}}_{M}^{C}\right\rangle \cap\left\langle\overline{\mathcal{M}}_{C}^{C}\right\rangle=\emptyset$. As the redundancy in $\left\langle\overline{\mathcal{M}}_{M}^{C}\right\rangle$ is already dealt with, we can focus on $\left\langle\overline{\mathcal{M}}_{C}^{C}\right\rangle$ which may also be redundant. We start by writing

$$
\left\langle\overline{\mathcal{M}}_{C}^{C}\right\rangle=\left\{\overline{\mathcal{M}}_{C}^{C}\right\}+\left\langle\overline{\mathcal{M}}_{\nu}^{C}\right\rangle .
$$

Here, $\left\{\overline{\mathcal{M}}_{C}^{C}\right\}$ is the linearly independent part which restricts the motion of the end effector to $\overline{\mathcal{M}}_{E}$, while $\left\langle\overline{\mathcal{M}}_{\nu}^{C}\right\rangle$ is the collection of the constraints that are linearly dependent of the entries in $\left\{\overline{\mathcal{M}}_{C}^{C}\right\}$. Thus, the redundant constraints given by the term $\left\langle\overline{\mathcal{M}}_{C}^{C}\right\rangle$ in Equation (31) are given by $\operatorname{card}\left\langle\overline{\mathcal{M}}_{\nu}^{C}\right\rangle$. The total redundancy in the constraint system is given by

$$
\operatorname{card}\left\langle\overline{\mathcal{M}}_{E \nu}^{C}\right\rangle=(k-1) \operatorname{card}\left\{\overline{\mathcal{M}}_{M}^{C}\right\}+\operatorname{card}\left\langle\overline{\mathcal{M}}_{\nu}^{C}\right\rangle .
$$

Finally, we also note that $\left\langle\overline{\mathcal{M}}_{E \nu}^{C}\right\rangle$ can also be factorised out from

$$
\left\langle\overline{\mathcal{M}}_{E}^{C}\right\rangle=\left\{\overline{\mathcal{M}}_{E}^{C}\right\}+\left\langle\overline{\mathcal{M}}_{E \nu}^{C}\right\rangle .
$$

\subsection{The Modified Grübler Formula}

The Grübler formula does not take redundant constraints into consideration. Redundant constraint are constraints that do not reduce the mobility of the end effector or the chains. We therefore need to add these to the Grübler formula. The Modified Grübler formula as presented in Dai et al. (2006) is given by adding (33) to $(18)$

$D=d N-\sum_{i=1}^{n}\left(d-f_{i}\right)+(k-1) \cdot \operatorname{card}\left\{\overline{\mathcal{M}}_{M}^{C}\right\}+\operatorname{card}\left\langle\overline{\mathcal{M}}_{\nu}^{C}\right\rangle$ 
where $d$ is the dimension of the space, normally 3 or 6 . This expression identifies the redundant constraints. It also gives the mobility due to self-motion. It does not, however, identify very easily due to what joints these motions occur. This is considered in more detail in the next section.

\subsection{The Motion Space}

The mobility of the mechanism tells us how many active joints are needed for the mechanism to be equilibrated. However, it does not tell us what joints can be set as passive and what joints need to be active. In the following, we will show that an alternative to the Modified Grübler formula given in (35) can be found from the motion space and we will show how this approach naturally leads to the classification of different types of overconstrained joints. This is the same classification of overconstrained chains as in Rico et al. (2003) and Rico et al. (2006). Further, in the next section, we will use this to set up a set of simple rules on where the active joints need to be placed, i.e. how many active joints need to be placed in each chain, as well as their position in the chain.

\subsubsection{Exceptional Linkages}

In Rico et al. (2003), two sub-chains that have an intersection, but for which the motion space of one subchain is not a subspace of the other, is denoted exceptional linkage. We refer to the work of Hervé (1978) for a formal definition of exceptional, trivial and paradoxical linkages. Paradoxical linkages are not treated here. In Rico et al. (2006) this is generalised to the case of arbitrarily many chains. Here we will look at it from a different view in order to get a deeper understanding of the mobility criterion.

We start by finding the mobility of the end effector. This is given by the intersection of the motion space of each chain

$$
\left\{\overline{\mathcal{M}}_{E}\right\}=\bigcap_{j=1}^{k}\left\{\overline{\mathcal{M}}_{j}\right\}
$$

evaluated at $g \in \mathcal{M}_{1} \cap \cdots \cap \mathcal{M}_{k}$. Thus the mobility of the end effector is given by $\operatorname{card}\left\{\overline{\mathcal{M}}_{E}\right\}$. Each chain may also have a mobility independent of the mobility of the end effector. This is the self-motion and is given by the degree of freedom in the chain and subtracting the dimension of the open loop end-effector motion of the chain.

$$
\begin{aligned}
\operatorname{card}\left\{\overline{\mathcal{M}}_{s m}\right\}_{j} & =\sum_{i=1}^{n_{j}} f_{i}-\operatorname{card}\left\{\overline{\mathcal{M}}_{j}\right\}, \\
\operatorname{card}\left\{\overline{\mathcal{M}}_{s m}\right\} & =\sum_{j=1}^{k}\left(\sum_{i=1}^{n_{j}} f_{i}-\operatorname{card}\left\{\overline{\mathcal{M}}_{j}\right\}\right) .
\end{aligned}
$$

The total mobility of the mechanism is then given by adding (37) to (36). We will write this as a proposition and provide a different proof than that of Rico et al. (2006).

Proposition 4.1. The total mobility of a mechanism is given by the degree of freedom of the end effector, given in Equation (36) and the self-motion of the chain, given in Equation (37) by

$$
\begin{aligned}
D & =\operatorname{card}\left\{\overline{\mathcal{M}}_{E}\right\}+\sum_{j=1}^{k}\left(\sum_{i=1}^{n_{j}} f_{i}-\operatorname{card}\left\{\overline{\mathcal{M}}_{j}\right\}\right), \\
& =\operatorname{card}\left\{\overline{\mathcal{M}}_{E}\right\}+\sum_{i=1}^{n} f_{i}-\sum_{j=1}^{k} \operatorname{card}\left\{\overline{\mathcal{M}}_{j}\right\} .
\end{aligned}
$$

Proof. (sketch) The result follows directly from the observations that a) the degree of freedom of the end effector is given by the dimension of $\left\{\overline{\mathcal{M}}_{E}\right\}$ in Equation (36); and b) that the self-motion of each chain is given by Equation (37).

a) The degree of freedom of the end effector is given by the dimension of $\left\{\overline{\mathcal{M}}_{E}\right\}$ in Equation (36). This follows directly from Meng et al. (2007).

b) The dimension of the self-motion manifold can be found in most textbooks on robotics (e.g. Murray et al. (1994)) to be the dimension of the null of the Jacobian $\mathcal{N}(J)$, which is given as $\operatorname{dim}(\mathcal{N}(J))=$ $n_{j}-m$ where $n_{j}$ is the number of joints and $m$ is the dimension of the end-effector motion for the open chain. This equivalent to (37).

We are mainly concerned with the effect of adding a joint to the chain. Adding a joint to a chain $\mathcal{M}$ will have the same effect as making one joint passive when only the passive joints of the manipulator are considered, i.e. adding a joint to $\mathcal{M}_{P}$. In this section we look at the effect of adding a joint to the mechanisms and in the next section we use these results to analyse in what case the manipulator is equilibrated. We will use the reasoning in Proposition 4.1 and the observation that a joint that increases the dimension of the end effector motion of the open chain, but not of the closed chain, will always be locked. Then there are three different outcomes of adding a joint to the manipulator: 
- $\sum_{i=1}^{n_{j}} f_{i}$ increases by one while $\operatorname{card}\left\{\overline{\mathcal{M}}_{j}\right\}$ does not.

- The dimension of the self-motion manifold of the chain increases by one.

- $\sum_{i=1}^{n_{j}} f_{i}$ and $\operatorname{card}\left\{\overline{\mathcal{M}}_{j}\right\}$ increase by one while $\operatorname{card}\left\{\overline{\mathcal{M}}_{E}\right\}$ does not.

- The joint will be locked and the mobility of the system does not change.

- $\sum_{i=1}^{n_{j}} f_{i}, \operatorname{card}\left\{\overline{\mathcal{M}}_{j}\right\}$ and $\operatorname{card}\left\{\overline{\mathcal{M}}_{E}\right\}$ increase by one.

- The mobility of the end effector increases by one.

- The dimension of the self-motion manifold of the chain does not increase.

Thus, by checking the dimension of $\operatorname{card}\left\{\overline{\mathcal{M}}_{j}\right\}$ and $\operatorname{card}\left\{\overline{\mathcal{M}}_{E}\right\}$ we can effectively find the effect that a joint failure has on the mobility of the end effector.

\subsubsection{Trivial Linkage of Type I}

In Rico et al. (2006) the case when all the sub-chains generate the same motion

$$
\left\{\overline{\mathcal{M}}_{j}\right\}=\left\{\overline{\mathcal{M}}_{E}\right\} \quad \text { for } j=1 \ldots k
$$

is denoted trivial linkages. In this case the mobility is found directly from (38) by

$$
\begin{aligned}
D & =\operatorname{card}\left\{\overline{\mathcal{M}}_{E}\right\}+\sum_{j=1}^{k}\left(\sum_{i=1}^{n_{j}} f_{i}-\operatorname{card}\left\{\overline{\mathcal{M}}_{E}\right\}\right) \\
& =\operatorname{card}\left\{\overline{\mathcal{M}}_{E}\right\}-k \cdot \operatorname{card}\left\{\overline{\mathcal{M}}_{E}\right\}+\sum_{j=1}^{k}\left(\sum_{i=1}^{n_{j}} f_{i}\right) \\
& =\sum_{i=1}^{n} f_{i}-(k-1) \cdot \operatorname{card}\left\{\overline{\mathcal{M}}_{E}\right\} .
\end{aligned}
$$

In this case there are only two different outcomes:

- $\sum_{i=1}^{n_{j}} f_{i}$ increases by one while $\operatorname{card}\left\{\overline{\mathcal{M}}_{j}\right\}$ does not.

- The dimension of the self-motion manifold of the chain increases by one.

- $\sum_{i=1}^{n_{j}} f_{i}$ and $\operatorname{card}\left\{\overline{\mathcal{M}}_{j}\right\}$ increase by one.

- The joint will be locked and the mobility of the system does not change.

- $\sum_{i=1}^{n_{j}} f_{i}, \operatorname{card}\left\{\overline{\mathcal{M}}_{j}\right\}$ and $\operatorname{card}\left\{\overline{\mathcal{M}}_{E}\right\}$ increase by one.

- Will never occur.

\subsubsection{Trivial Linkage of Type II}

In Rico et al. (2003) the case when the entire motion of the end-effector, connected by two sub-chains, can be determined and is restricted by one chain is denoted trivial linkage. As they only consider single loops, the constraints of the other chain does not affect the mobility of the end effector. In our setting, we define the corresponding multi-loop classification of trivial linkage as the following. Assume that we have $E^{\prime}$ manipulators that all generate $\overline{\mathcal{M}}_{E}$ and $M^{\prime}$ manipulators that all generate $\overline{\mathcal{M}}_{M}$, where $\overline{\mathcal{M}}_{E} \subset \overline{\mathcal{M}}_{M}$. Then the total mobility of the system is given by $\overline{\mathcal{M}}_{E}$ and the internal mobility of each of the chains. The internal mobility of the chains in $\mathcal{M}_{E}$ and $\mathcal{M}_{M}$ must, however, be treated differently.

The total mobility of the system is then given by

$$
\begin{aligned}
D= & \operatorname{card}\left\{\overline{\mathcal{M}}_{E}\right\}+\sum_{j=1}^{k}\left(\sum_{i=1}^{n_{j}} f_{i}-\operatorname{card}\left\{\overline{\mathcal{M}}_{j}\right\}\right) \\
= & \operatorname{card}\left\{\overline{\mathcal{M}}_{E}\right\}+\sum_{E}\left(\sum_{i=1}^{n_{j}} f_{i}-\operatorname{card}\left\{\overline{\mathcal{M}}_{E}\right\}\right) \\
& +\sum_{M}\left(\sum_{i=1}^{n_{j}} f_{i}-\operatorname{card}\left\{\overline{\mathcal{M}}_{M}\right\}\right) \\
= & \sum_{i=1}^{n} f_{i}-\left(E^{\prime}-1\right) \cdot \operatorname{card}\left\{\overline{\mathcal{M}}_{E}\right\}-M^{\prime} \cdot \operatorname{card}\left\{\overline{\mathcal{M}}_{M}\right\}
\end{aligned}
$$

where $\sum_{E}$ sums over all the chains that generate $\overline{\mathcal{M}}_{E}$ and $\sum_{M}$ sums over all the chains that generate $\overline{\mathcal{M}}_{M}$. Also in this case there are three different outcomes which we will divide into two classes:

For $j \in M$,

- $\sum_{i=1}^{n_{j}} f_{i}$ increases by one while $\operatorname{card}\left\{\overline{\mathcal{M}}_{j}\right\}$ does not.

- The dimension of the self-motion manifold of the chain increases by one.

- $\sum_{i=1}^{n_{j}} f_{i}$ and $\operatorname{card}\left\{\overline{\mathcal{M}}_{j}\right\}$ increase by one while $\operatorname{card}\left\{\overline{\mathcal{M}}_{E}\right\}$ does not.

- The joint will be locked and the mobility of the system does not change.

- $\sum_{i=1}^{n_{j}} f_{i}, \operatorname{card}\left\{\overline{\mathcal{M}}_{M}\right\}$ and $\operatorname{card}\left\{\overline{\mathcal{M}}_{E}\right\}$ increase by one.

- Will never occur.

For $j \in E$

- $\sum_{i=1}^{n_{j}} f_{i}$ increases by one while $\operatorname{card}\left\{\overline{\mathcal{M}}_{j}\right\}$ does not.

- The dimension of the self-motion manifold of the chain increases by one. 
- $\sum_{i=1}^{n_{j}} \frac{f_{i}}{\mathcal{M}_{E}}$ and $\operatorname{card}\left\{\overline{\mathcal{M}}_{j}\right\}$ increase by one while $\operatorname{card}\left\{\overline{\mathcal{M}}_{E}\right\}$ does not.

- The joint will be locked and the mobility of the system does not change.

- $\sum_{i=1}^{n_{j}} f_{i}, \operatorname{card}\left\{\overline{\mathcal{M}}_{M}\right\}$ and $\operatorname{card}\left\{\overline{\mathcal{M}}_{E}\right\}$ increase by one.

- The mobility of the end effector increases by one. The self-motion of the chain does not increase.

\section{Fault Tolerance}

In this section, we look into the effect of free-swinging joint failure (FSJF), or torque failure, in parallel manipulators and how the results from the previous section can be used to prevent that the mechanism turns inequilibrated when this occurs. For a general treatment and an approach on how to identify joint failure see Tinós et al. (2006). In this case, as the number of passive joints in the manipulator increases by one, the mobility of $\mathcal{M}_{P}$ may remain zero or increase by one. Let $m$ be the number of active joints in $\mathcal{M}$. When $\mathcal{M}_{P}$ does not allow any motion after the joint failure, we have

$$
D_{m}=0 \stackrel{F S J F}{\rightleftharpoons} D_{m-1}=0
$$

and the manipulator remains equilibrated with respect to external forces. When $\mathcal{M}_{P}$ allows a 1 DOF motion as a result of the joint failure, i.e.

$$
D_{m}=0 \stackrel{\text { FSJF }}{\rightleftharpoons} D_{m-1}=1,
$$

the mechanism is not fault tolerant.

We are interested in the condition for which $D_{m-1}=$ 0 . As seen in the previous section, the effect of a freeswinging joint failure depends on the joint. We start by setting up a set of rules that determines if a joint failure will increase the mobility of $\mathcal{M}_{P}$. This can also be used as a design criterion to guarantee the mechanism to be fault tolerant. We do that by determining where to put the actuator redundancy most effectively in order for the manipulator to be resistant to a joint failure of any joint.

In the following we will find the conditions for which the mechanism is equilibrated for all the different outcomes of joint failure found in Section 4.3.

\subsubsection{Exceptional Linkages}

- $\sum_{i=1}^{n_{j}} f_{i}$ increases by one while $\operatorname{card}\left\{\overline{\mathcal{M}}_{j}\right\}$ does not.

- The end effector is equilibrated. Chain $j$ will only remain equilibrated if it is actuator redundant. The redundancy must be in the set of joints in which the self-motion occurs.
- $\sum_{i=1}^{n_{j}} \frac{f_{i}}{\mathcal{M}_{E}}$ and $\operatorname{card}\left\{\overline{\mathcal{M}}_{j}\right\}$ increase by one while $\operatorname{card}\left\{\overline{\mathcal{M}}_{E}\right\}$ does not.

- No action needed. Both end effector and chains are equilibrated.

- $\sum_{i=1}^{n_{j}} f_{i}, \operatorname{card}\left\{\overline{\mathcal{M}}_{j}\right\}$ and $\operatorname{card}\left\{\overline{\mathcal{M}}_{E}\right\}$ increase by one.

- The mobility can be compensated with actuator redundancy in any joint that is not locked for the motion generated by $\mathcal{M}_{P}$.

\subsubsection{Trivial Linkage of Type I}

- $\sum_{i=1}^{n_{j}} f_{i}$ increases by one while $\operatorname{card}\left\{\overline{\mathcal{M}}_{j}\right\}$ does not.

- The end effector is equilibrated. Chain $j$ will only remain equilibrated if it is actuator redundant. The redundancy must be in the set of joints in which the self-motion occurs.

- $\sum_{i=1}^{n_{j}} f_{i}$ and $\operatorname{card}\left\{\overline{\mathcal{M}}_{j}\right\}$ increase by one.

- No action needed. Both end effector and chains are equilibrated.

- $\sum_{i=1}^{n_{j}} f_{i}, \operatorname{card}\left\{\overline{\mathcal{M}}_{j}\right\}$ and $\operatorname{card}\left\{\overline{\mathcal{M}}_{E}\right\}$ increase by one.

- Will never occur.

\subsubsection{Trivial Linkage of Type II}

For $j \in M$,

- $\sum_{i=1}^{n_{j}} f_{i}$ increases by one while $\operatorname{card}\left\{\overline{\mathcal{M}}_{j}\right\}$ does not.

- The end effector is equilibrated. Chain $j$ will only remain equilibrated if it is actuator redundant. The redundancy must be in the set of joints in which the self-motion occurs.

- $\sum_{i=1}^{n_{j}} \frac{f_{i}}{\mathcal{M}_{E}}$ and $\operatorname{card}\left\{\overline{\mathcal{M}}_{j}\right\}$ increase by one while $\operatorname{card}\left\{\overline{\mathcal{M}}_{E}\right\}$ does not.

- No action needed. Both end effector and chains are equilibrated.

- $\sum_{i=1}^{n_{j}} f_{i}, \operatorname{card}\left\{\overline{\mathcal{M}}_{M}\right\}$ and $\operatorname{card}\left\{\overline{\mathcal{M}}_{E}\right\}$ increase by one.

- Will never occur.

For $j \in E$,

- $\sum_{i=1}^{n_{j}} f_{i}$ increases by one while $\operatorname{card}\left\{\overline{\mathcal{M}}_{j}\right\}$ does not. 
- The end effector is equilibrated. Chain $j$ will only remain equilibrated if it is actuator redundant. The redundancy must be in the set of joints in which the self-motion occurs.

- $\sum_{i=1}^{n_{j}} \frac{f_{i}}{\mathcal{M}_{E}}$ and $\operatorname{card}\left\{\overline{\mathcal{M}}_{j}\right\}$ increase by one while $\operatorname{card}\left\{\overline{\mathcal{M}}_{E}\right\}$ does not.

- No action needed. Both end effector and chains are equilibrated.

- $\sum_{i=1}^{n_{j}} f_{i}, \operatorname{card}\left\{\overline{\mathcal{M}}_{M}\right\}$ and $\operatorname{card}\left\{\overline{\mathcal{M}}_{E}\right\}$ increase by one.

- The mobility can be compensated with actuator redundancy in any joint that is not locked for the motion generated by $\mathcal{M}_{P}$.

From the results presented in this section, we see that we can easily verify if redundant actuation is needed when joint failure occurs for a given active joint. If joint failure does not lead to self-motion of the chain it may be compensated for by another redundant actuated joint that is not locked for this motion. These cases are important to recognise in order to not place unnecessary many active joints in the mechanism. If, on the other hand, the joint failure leads to self-motion, a redundant actuated joint is always needed in the respective chain. These observations lead to a simple rule on how to place the redundant active joints in order to guarantee that the mechanism remains equilibrated when actuator failure occurs for an arbitrary joint.

\section{Examples}

We are interested in the condition for which $D_{m-1}=0$. As seen in the previous section, the effect of a free swinging joint failure depends on the joint in which it occurs. In the following we show three examples on how to determine where to put the actuator redundancy most effectively in order guarantee fault tolerance with respect to any joint.

\subsection{Exceptional Linkages}

Consider the mechanisms in Figure 1. One of the arms is kinematically redundant in order to avoid obstacles. We find the twists representing each chain

$$
\begin{aligned}
\overline{\mathcal{M}}_{1}= & \left\{\left[\begin{array}{c}
v_{y} \\
0
\end{array}\right],\left[\begin{array}{c}
p_{12} \times w_{y} \\
w_{y}
\end{array}\right],\left[\begin{array}{c}
p_{13} \times w_{y} \\
w_{y}
\end{array}\right],\left[\begin{array}{c}
p_{14} \times w_{y} \\
w_{y}
\end{array}\right]\right\}, \\
\overline{\mathcal{M}}_{2}= & \left\{\left[\begin{array}{c}
v_{z} \\
0
\end{array}\right],\left[\begin{array}{c}
p_{22} \times w_{z} \\
w_{z}
\end{array}\right],\left[\begin{array}{c}
p_{23} \times w_{z} \\
w_{z}
\end{array}\right],\left[\begin{array}{c}
p_{24} \times w_{z} \\
w_{z}
\end{array}\right]\right. \\
& {\left.\left[\begin{array}{c}
p_{25} \times w_{z} \\
w_{z}
\end{array}\right], \operatorname{Ad}_{g_{2, b 5}}\left[\begin{array}{c}
p_{26} \times w_{x} \\
w_{x}
\end{array}\right]\right\} . }
\end{aligned}
$$

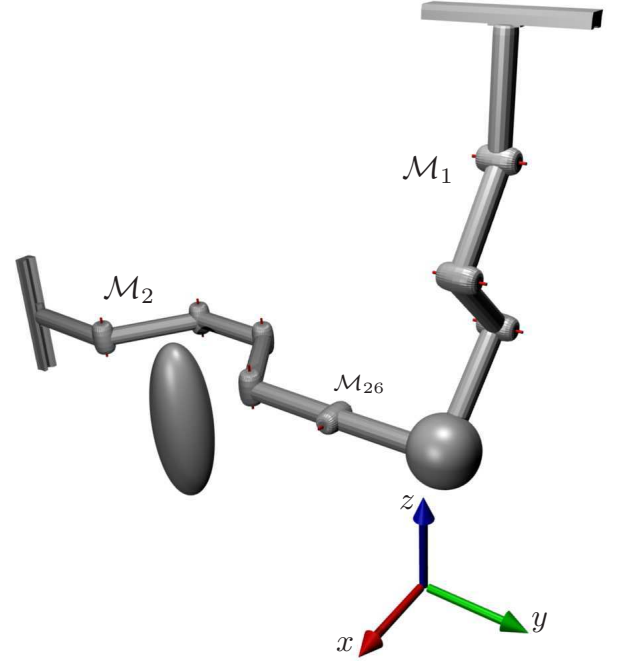

Figure 1: Exceptional linkage with self motion

where $p_{j i}=\left[\begin{array}{lll}x_{j i} & y_{j i} & z_{j i}\end{array}\right]^{\top}$ is some point on the revolute axis of joint $i$ in chain $j$ given in the interial frame. We can write the transformation from the base $b$ to joint 5 of $\mathcal{M}_{2}$ as $R_{2, b 5}$ and $p_{2, b 5}$ given by

$$
\begin{aligned}
& R_{2, b 5}\left(\theta_{2(2-5)}\right)=\left[\begin{array}{ccc}
\cos \theta_{2} & -\sin \theta_{2} & 0 \\
\sin \theta_{2} & \cos \theta_{2} & 0 \\
0 & 0 & 1
\end{array}\right] \\
& p_{2, b 5}\left(\theta_{1(1-5)}\right)=\left[\begin{array}{lll}
x_{2, b 4} & y_{2, b 4} & z_{2, b 4}
\end{array}\right]^{\top}
\end{aligned}
$$

where $\theta_{2}=\sum_{i=1}^{4} \theta_{2 i}$. We can then write the twists as

$$
\overline{\mathcal{M}}_{1}=\left\{\left[\begin{array}{l}
0 \\
1 \\
0 \\
0 \\
0 \\
0
\end{array}\right],\left[\begin{array}{c}
z_{12} \\
0 \\
-x_{12} \\
0 \\
1 \\
0
\end{array}\right],\left[\begin{array}{c}
z_{13} \\
0 \\
-x_{13} \\
0 \\
1 \\
0
\end{array}\right],\left[\begin{array}{c}
z_{14} \\
0 \\
-x_{14} \\
0 \\
1 \\
0
\end{array}\right]\right\},
$$

$$
\overline{\mathcal{M}}_{2}=\left\{\left[\begin{array}{l}
0 \\
0 \\
1 \\
0 \\
0 \\
0
\end{array}\right],\left[\begin{array}{c}
y_{22} \\
-x_{22} \\
0 \\
0 \\
0 \\
1
\end{array}\right],\left[\begin{array}{c}
y_{23} \\
-x_{23} \\
0 \\
0 \\
0 \\
1
\end{array}\right],\left[\begin{array}{c}
y_{24} \\
-x_{24} \\
0 \\
0 \\
0 \\
1
\end{array}\right],\right.
$$

$$
\left.\left[\begin{array}{c}
y_{25} \\
-x_{25} \\
0 \\
0 \\
0 \\
1
\end{array}\right],\left[\begin{array}{c}
-z_{26} z_{2, b 4} \cos \theta_{2}-y_{26} y_{2, b 4} \\
-z_{26} z_{2, b 4} \sin \theta_{2}+y_{26} x_{2, b 4} \\
z_{26} y_{2, b 4} \sin \theta_{2}-z_{26} x_{2, b 4} \cos \theta_{2} \\
\cos \theta_{3} \\
\sin \theta_{3} \\
0
\end{array}\right]\right\}
$$




\subsubsection{Equilibrated Mechanism}

The motion of each chain is given by

$$
\overline{\mathcal{M}}_{1} \in \mathcal{X}(y), \quad \overline{\mathcal{M}}_{2} \in \mathcal{X}(z) \times \mathcal{R}\left(w_{6}\right)
$$

where $\mathcal{X}(w)$ is the Schoenflies group (3D translation and rotation about $w$ ) and

$$
w_{6}=R_{2, b 5} w_{x} .
$$

The constrained space is given by

$$
\begin{aligned}
& \overline{\mathcal{M}}_{1}^{C}=\left\{\left[\begin{array}{c}
0 \\
w_{x}
\end{array}\right],\left[\begin{array}{c}
0 \\
w_{z}
\end{array}\right]\right\} \\
& \overline{\mathcal{M}}_{2}^{C}=\left\{\left[\begin{array}{llllll}
0 & 0 & 0 & -\sin \theta_{3} & \cos \theta_{3} & 0
\end{array}\right]\right\}^{\top} \\
& =\left\{\left[\begin{array}{c}
0 \\
R_{2, b 5} w_{x}
\end{array}\right]\right\} \text {. }
\end{aligned}
$$

Due to the kinematic constraints we have $\theta_{2}=0$ which gives us

$$
\overline{\mathcal{M}}_{E}^{C}=\overline{\mathcal{M}}_{2}^{C} \cup \overline{\mathcal{M}}_{1}^{C}=\left\{\left[\begin{array}{c}
0 \\
w_{x}
\end{array}\right],\left[\begin{array}{c}
0 \\
w_{y}
\end{array}\right],\left[\begin{array}{c}
0 \\
w_{z}
\end{array}\right]\right\} .
$$

Note that none of these are redundant. We can now write

$$
\overline{\mathcal{M}}_{E}=\left(\overline{\mathcal{M}}_{E}^{C}\right)^{\perp}=\left\{\left[\begin{array}{c}
w_{x} \\
0
\end{array}\right],\left[\begin{array}{c}
w_{y} \\
0
\end{array}\right],\left[\begin{array}{c}
w_{z} \\
0
\end{array}\right]\right\} \in \mathcal{T}(3) .
$$

These are clearly multisets, i.e. there are several different combinations of joints that generate the particular motion. We can now verify that the mechanism is exceptional, i.e.

$$
\begin{array}{ll}
\overline{\mathcal{M}}_{E} \subset \overline{\mathcal{M}}_{1}, & \overline{\mathcal{M}}_{E} \subset \overline{\mathcal{M}}_{2}, \\
\overline{\mathcal{M}}_{1} \nsubseteq \overline{\mathcal{M}}_{2}, & \overline{\mathcal{M}}_{2} \nsubseteq \overline{\mathcal{M}}_{1} .
\end{array}
$$

As the end effector has three degrees of freedom, we need at least three active joints. These can be chosen arbitrarily among the joints that generate the endeffector motion, i.e. all joints except $\mathcal{M}_{26}$. At this stage it is important that all the degrees of freedom in (53) and the internal motion are taken care of. To see this we look at Equation (38) where we see that the mobility can be divided into the degree of freedom of the end-effector and the self-motion of the chains

$$
D=\underbrace{\operatorname{card}\left\{\overline{\mathcal{M}}_{E}\right\}}_{\text {End-effector DOF }}+\sum_{j=1}^{k} \underbrace{\left(\sum_{i=1}^{n_{j}} f_{i}-\operatorname{card}\left\{\overline{\mathcal{M}}_{j}\right\}\right)}_{\text {DOF of self-motion of chain } j} .
$$

We get the following set of rules for choosing the joints;

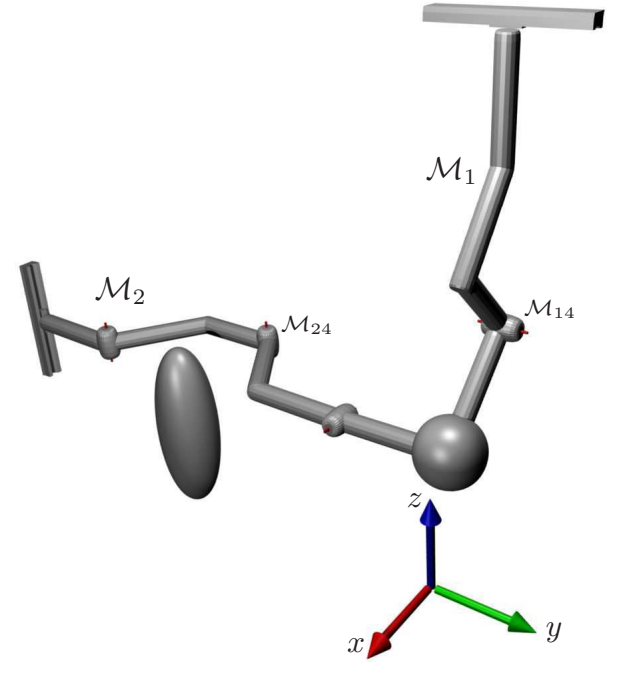

Figure 2: Exceptional linkage. We consider the active joints as fixed and consider only the passive joints, i.e. $\mathcal{M}_{P}$. The mechanism $\mathcal{M}_{P}$ is equilibrated with respect to any external disturbance. If $\mathcal{M}_{24}$ is chosen as the redundant actuation, the manipulator is also fault tolerant. If $\mathcal{M}_{14}$ is chosen, it is not fault tolerant

- choose four active joint of which at least one is chosen among the joints that generate the self motion,

- choose the joints so that they generate the endeffector motion $\mathcal{T}(3)$.

One example of how the active joints can be chosen is shown in Figure 2. We illustrate the mobility of $\mathcal{M}_{P}$ by setting the active joints rigid. We see that the manipulator, considering passive joints only, is now equilibrated with respect to any external disturbances.

\subsubsection{Fault tolerance}

Assume we have chosen a set of joints like the ones in Figure 2 for which the manipulator is equilibrated. After observing that $\mathcal{M}_{26}$ does not affect the end-effector or internal motion we get the twists

$$
\begin{aligned}
& \overline{\mathcal{M}}_{P 1}=\left\{\left[\begin{array}{c}
v_{y} \\
0
\end{array}\right],\left[\begin{array}{c}
p_{14} \times w_{y} \\
w_{y}
\end{array}\right]\right\}, \\
& \overline{\mathcal{M}}_{P 2}=\left\{\left[\begin{array}{c}
v_{z} \\
0
\end{array}\right],\left[\begin{array}{c}
p_{22} \times w_{z} \\
w_{z}
\end{array}\right],\left[\begin{array}{c}
p_{24} \times w_{z} \\
w_{z}
\end{array}\right]\right\} .
\end{aligned}
$$

Then the effect of adding a joint to the chain in $\mathcal{M}_{P}$, i.e. turning one of the active joints passive in $\mathcal{M}$, depends on the joint in question. We first note that

$$
\overline{\mathcal{M}}_{P}=\overline{\mathcal{M}}_{P 1} \cap \overline{\mathcal{M}}_{P 2}=\emptyset .
$$


We need to choose one or more additional joints in order to guarantee that the manipulator remains equilibrated when joint failure occurs. We will look into two different cases. First we choose $\mathcal{M}_{14}$ to be the redundant active joint, then we chose $\mathcal{M}_{22}$ (or equivalently $\mathcal{M}_{24}$ ) and we will see how important this choice is for fault tolerance of the mechanism.

Assume fist we choose $\mathcal{M}_{14}$ as the redundant active joint (see Figure 2). We then have two different cases that need to be considered. See From and Gravdahl (2009) for a detailed analysis on the effect of joint failure.

- One of the active joints in $\mathcal{M}_{1}$ becomes passive:

- $\sum_{\mathcal{M}_{P 1}} f_{i}$ and $\operatorname{card}\left\{\overline{\mathcal{M}}_{P 1}\right\}$ increase by one while card $\left\{\overline{\mathcal{M}}_{P}\right\}$ does not. $\mathcal{M}$ remains equilibrated.

$$
D_{5}=0 \stackrel{F S J F}{\rightleftharpoons} D_{4}=0 .
$$

- One of the active joints in $\mathcal{M}_{2}$ becomes passive:

- $\sum_{\mathcal{M}_{P 2}} f_{i}, \operatorname{card}\left\{\overline{\mathcal{M}}_{P 2}\right\}$ and $\operatorname{card}\left\{\overline{\mathcal{M}}_{P}\right\}$ increase by one. The end effector of $\mathcal{M}_{P}$ has one degree of freedom $(\mathcal{T}(y))$.

$$
D_{5}=0 \stackrel{F S J F}{\rightleftharpoons} D_{4}=1 .
$$

We can see this if we write out the twists of $\mathcal{M}_{P}$ when $\mathcal{M}_{14}$ is chosen as the redundant actuation and joint failure occurs in $\mathcal{M}_{23}$ :

$$
\begin{aligned}
& \overline{\mathcal{M}}_{P 1}=\left\{\left[\begin{array}{c}
v_{y} \\
0
\end{array}\right]\right\}, \\
& \overline{\mathcal{M}}_{P 2}=\left\{\left[\begin{array}{c}
v_{z} \\
0
\end{array}\right],\left[\begin{array}{c}
p_{22} \times w_{z} \\
w_{z}
\end{array}\right],\left[\begin{array}{c}
p_{23} \times w_{z} \\
w_{z}
\end{array}\right],\left[\begin{array}{c}
p_{24} \times w_{z} \\
w_{z}
\end{array}\right]\right\} .
\end{aligned}
$$

and

$$
\overline{\mathcal{M}}_{P}=\overline{\mathcal{M}}_{P 1} \cap \overline{\mathcal{M}}_{P 2}=\mathcal{T}(y) .
$$

We see that when the actuator redundancy is chosen in $\mathcal{M}_{1}$ the mechanism is fault tolerant with respect to joint failures in $\mathcal{M}_{1}$ only.

For $\mathcal{M}_{22} / \mathcal{M}_{24}$ we also consider the same two cases, there is no difference if we choose $\mathcal{M}_{22}$ or $\mathcal{M}_{24}$.

- One of the active joints in $\mathcal{M}_{1}$ becomes passive:

- $\sum_{\mathcal{M}_{P 1}} f_{i}$ and $\operatorname{card}\left\{\overline{\mathcal{M}}_{P 1}\right\}$ increase by one while card $\left\{\overline{\mathcal{M}}_{P}\right\}$ does not. $\mathcal{M}$ remains equilibrated.

$$
D_{5}=0 \stackrel{F S J F}{\rightleftharpoons} D_{4}=0 .
$$

- One of the active joints in $\mathcal{M}_{2}$ becomes passive:

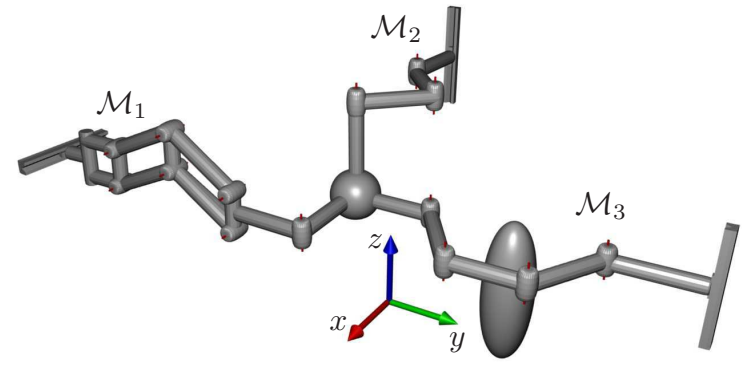

Figure 3: Trivial Linkage of Type I.

- $\sum_{M_{P 2}} f_{i}$ and $\operatorname{card}\left\{\overline{\mathcal{M}}_{2}\right\}$ increase by one while card $\left\{\overline{\mathcal{M}}_{P}\right\}$ does not. $\mathcal{M}$ remains equilibrated.

$$
D_{5}=0 \stackrel{F S J F}{=} D_{4}=0 .
$$

Again we write out the twists of $\mathcal{M}_{P}$ when $\mathcal{M}_{24}$ is chosen as the redundant actuation and joint failure occurs in $\mathcal{M}_{23}$ :

$$
\begin{aligned}
& \overline{\mathcal{M}}_{P 1}=\left\{\left[\begin{array}{c}
v_{y} \\
0
\end{array}\right],\left[\begin{array}{c}
p_{14} \times w_{y} \\
w_{y}
\end{array}\right]\right\}, \\
& \overline{\mathcal{M}}_{P 2}=\left\{\left[\begin{array}{c}
v_{z} \\
0
\end{array}\right],\left[\begin{array}{c}
p_{22} \times w_{z} \\
w_{z}
\end{array}\right],\left[\begin{array}{c}
p_{23} \times w_{z} \\
w_{z}
\end{array}\right]\right\} .
\end{aligned}
$$

and

$$
\overline{\mathcal{M}}_{P}=\overline{\mathcal{M}}_{P 1} \cap \overline{\mathcal{M}}_{P 2}=\emptyset .
$$

We see that when the actuator redundancy is chosen in $\mathcal{M}_{2}$ the mechanism is fault tolerant with respect to joint failures in all joints.

\subsection{Trivial Linkage of Type I}

Mechanisms for which all chains generate the same motion have some special characteristics. First of all, none of the joints are locked due to the kinematic constraints. Also, a joint fault in one chain which reduces the mobility of the end effector can be compensated for by actuator redundancy in any other chain. The chains may be kinematically redundant which is the case for $\mathcal{M}_{3}$ in Figure 3. As the reasoning is similar to the previous example, we give only a cursory description of how to choose the active joints.

\subsubsection{Equilibrated Mechanism}

As all chains generate the same motion we have more freedom in choosing the active joints. All the chains in Figure 3 generate $\mathcal{X}(z)$ which gives the end effector four degrees of freedom. $\mathcal{M}_{3}$ also has one degree of internal freedom. Hence, we need to choose at least one of the 


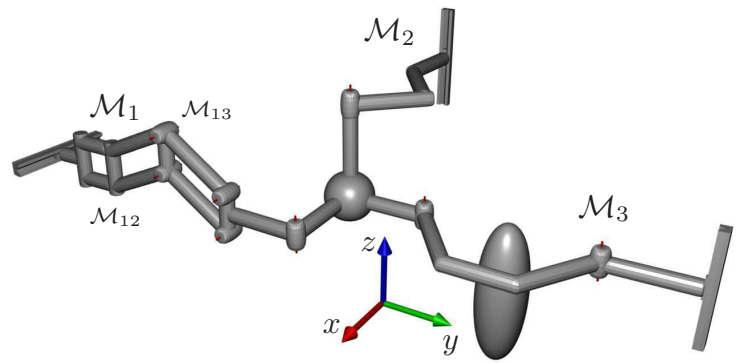

Figure 4: Trivial Linkage of Type I. Bad choice of active joints. If joint failure occurs in joint $\mathcal{M}_{12}$, the mechanism is no longer equilibrated with respect to forces in the direction of the $z$-axis, such as gravity forces. Joints $\mathcal{M}_{12}$ and $\mathcal{M}_{12}$ are parallelogram joints that generate motion in $S^{1}$.

joints in the set that generate the self-motion as active and the other three can be chosen arbitrarily as long as the other degrees of freedom $\left(\mathcal{X}(z) \backslash \mathcal{R}\left(z, p_{3 i}\right)\right)$ can be generated. ${ }^{2}$

\subsubsection{Fault Tolerance}

Assume that we follow the reasoning from the previous example and choose the active joints as in Figure 4 with $\mathcal{M}_{12}, \mathcal{M}_{22}, \mathcal{M}_{23}, \mathcal{M}_{33}$ and $\mathcal{M}_{34}$ active. The motivation for this choice is that internal motion is present in $\mathcal{M}_{3}$, so we need one redundant actuator among these joints $\left(\mathcal{M}_{33}\right.$ and $\left.\mathcal{M}_{34}\right)$. This redundant actuator will also assure fault tolerance when joint failure occurs in joints in the other chains that generate the same motion, i.e. the intersection of the motions generated by the redundant actuator and the motion of the fault tolerant joint is non-empty.

If, however, joint failure occurs in $\mathcal{M}_{12}$, chain $\mathcal{M}_{1}$ can generate a motion in the direction of the $z$-axis. We have

$$
\mathcal{T}(z) \cap \mathcal{M}_{3 i}=\emptyset, \quad \text { for } i=2,3,4,5 .
$$

As the intersection is empty, the redundancy in $\mathcal{M}_{3}$ does not make the mechanism fault tolerant with respect to joint $\mathcal{M}_{12}$. To guarantee fault tolerance with respect to all joints, we need actuator redundancy in the joints that generate motion in this direction as well. This can be obtained by joints $\mathcal{M}_{12}, \mathcal{M}_{21}$ or $\mathcal{M}_{31}$. We thus conclude that, for the mechanism to be fault tolerant we need two redundant actuated joints.

\footnotetext{
${ }^{2}$ Loosely speaking, $\mathcal{X}(z) \backslash \mathcal{R}\left(z, p_{3 i}\right)$ can be interpreted as the motion $\mathcal{X}(z)$ minus the motion of $\mathcal{R}\left(z, p_{3 i}\right)$, for a formal definition, see Meng et al. (2007). $\mathcal{R}(z, p)$ rotates the point $p$ around the axis $z$.
}

\subsection{Trivial Linkage of Type II}

Consider the manipulator in Figure 5. The chains are described by the twists

$$
\begin{aligned}
& \overline{\mathcal{M}}_{1}=\left\{\left[\begin{array}{c}
v_{y} \\
0
\end{array}\right]\right\}, \\
& \overline{\mathcal{M}}_{2}=\left\{\left[\begin{array}{c}
p_{21} \times w_{z} \\
w_{z}
\end{array}\right],\left[\begin{array}{c}
p_{22} \times w_{z} \\
w_{z}
\end{array}\right],\left[\begin{array}{c}
p_{23} \times w_{z} \\
w_{z}
\end{array}\right]\right\}, \\
& \overline{\mathcal{M}}_{3}=\left\{\left[\begin{array}{c}
p_{31} \times w_{x} \\
w_{x}
\end{array}\right],\left[\begin{array}{c}
p_{32} \times w_{x} \\
w_{x}
\end{array}\right],\left[\begin{array}{c}
p_{32} \times w_{x} \\
w_{x}
\end{array}\right],\right. \\
&\left.\left(\operatorname{Ad}_{g_{3, b 3}}\right)\left[\begin{array}{c}
p_{34} \times w_{z} \\
w_{z}
\end{array}\right],\left(\operatorname{Ad}_{g_{3, b 4}}\right)\left[\begin{array}{c}
p_{35} \times w_{z} \\
w_{z}
\end{array}\right]\right\} .
\end{aligned}
$$

Due to the kinematic constraints, $R_{3, b 3}$ and $R_{3, b 4}$ are constant and

$$
\begin{gathered}
\overline{\mathcal{M}}_{1} \in \mathcal{T}(y), \quad \overline{\mathcal{M}}_{2} \in \mathcal{P} \mathcal{L}(z), \\
\overline{\mathcal{M}}_{3} \in \mathcal{P} \mathcal{L}(x) \times \mathcal{R}\left(w_{4}\right) \times \mathcal{R}\left(w_{5}\right) .
\end{gathered}
$$

where

$$
w_{4}=R_{3,03} w_{z}, \quad w_{5}=R_{3,04} w_{z},
$$

so that $w_{4}=w_{5}$. We then have

$$
\begin{aligned}
& \overline{\mathcal{M}}_{1}^{C}=\left\{\left[\begin{array}{c}
v_{x} \\
0
\end{array}\right],\left[\begin{array}{c}
v_{z} \\
0
\end{array}\right],\left[\begin{array}{c}
0 \\
w_{x}
\end{array}\right],\left[\begin{array}{c}
0 \\
w_{y}
\end{array}\right],\left[\begin{array}{c}
0 \\
w_{z}
\end{array}\right]\right\}, \\
& \overline{\mathcal{M}}_{2}^{C}=\left\{\left[\begin{array}{c}
v_{z} \\
0
\end{array}\right],\left[\begin{array}{c}
0 \\
w_{y}
\end{array}\right],\left[\begin{array}{c}
0 \\
w_{z}
\end{array}\right]\right\}, \\
& \overline{\mathcal{M}}_{3}^{C}=\left\{\left[\begin{array}{c}
0 \\
R_{3,03} w_{y}
\end{array}\right]\right\} .
\end{aligned}
$$

Considering the kinematic constraint, the constraint space of $\mathcal{M}_{3}$ becomes simply $\overline{\mathcal{M}}_{3}^{C}=\left\{\left[\begin{array}{ll}0 & w_{y}\end{array}\right]^{\top}\right\}$. We see that

$$
\overline{\mathcal{M}}_{3}^{C} \subset \overline{\mathcal{M}}_{2}^{C} \subset \overline{\mathcal{M}}_{1}^{C}
$$

or alternatively

$$
\overline{\mathcal{M}}_{1} \subset \overline{\mathcal{M}}_{2} \subset \overline{\mathcal{M}}_{3}
$$

which is the definition of trivial linkage of type II. This expression can be seen from (64) when applying the kinematic constraints.

\subsubsection{Equilibrated Mechanism}

The motion of the end effector is given by

$$
\overline{\mathcal{M}}_{E}=\overline{\mathcal{M}}_{1} \cap \overline{\mathcal{M}}_{2} \cap \overline{\mathcal{M}}_{3}=\overline{\mathcal{M}}_{1}=\mathcal{T}(y)
$$

and has thus only one degree of freedom. $\mathcal{M}_{2}$ has no internal motion, as can be seen from analysing $\overline{\mathcal{M}}_{1}$ and $\overline{\mathcal{M}}_{2}$ in the plane. $\mathcal{M}_{1} \cup \mathcal{M}_{2}$ has only one degree of freedom, which is the same as the end effector, and has no 


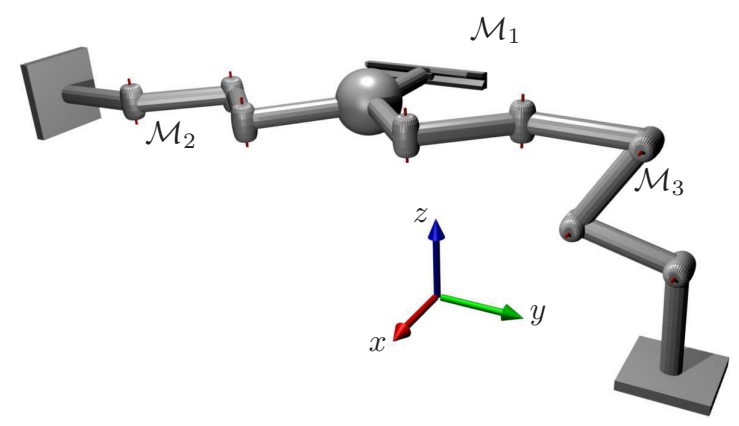

Figure 5: Trivial linkage of type II

self-motion. We can also use (21) (non-overconstrained mechanisms) with $d=6, N=4, n=5$ and $f_{i}=1$ to see that $\mathcal{M}_{3}$ allows no internal motion.

As the mechanism has mobility one, we need one active joint to make the manipulator equilibrated. This joint must generate the motion $\mathcal{T}(y)$. From (64) it is clear that all the joints in $\mathcal{M}_{1}$ and $\mathcal{M}_{2}$ generate the end-effector motion. If we take a close look at $\mathcal{M}_{3}$, we see that the three first joints generate $\mathcal{P} \mathcal{L}(x)$. The last two joints in this chain generate $\mathcal{R}\left(w_{4}\right) \times \mathcal{R}\left(w_{5}\right)$. This motion depends on the configuration of the first three joints. Due to the joint constraints we have $\theta_{31}+\theta_{32}+$ $\theta_{33}=0$ and thus $w_{4}=w_{5}=w_{z}$. As

$$
\mathcal{T}(y) \cap\left(\mathcal{R}\left(p_{34}, w_{z}\right) \times \mathcal{R}\left(p_{35}, w_{z}\right)\right)=\emptyset
$$

we conclude that these joints are locked.

To guarantee that the mechanism is equilibrated we can choose any joint that generate the end-effector motion. The only joints that do not generate this motion is $\mathcal{M}_{34}$ and $\mathcal{M}_{35}$. These joints will always be locked. The mechanism is equilibrated whenever any of the other joints are actuated.

\subsubsection{Fault tolerance}

The same applies to fault tolerance. To guarantee that the manipulator remains equilibrated when joint failure occurs we can choose any joint that generate the endeffector motion as the redundant actuation.

\section{Conclusion}

A set of rules on how to place redundant actuators in parallel mechanisms in order to guarantee that the manipulator remains equilibrated when actuator failure occurs is presented. The manipulator is said to be equilibrated when the manipulator, considering the passive joints only, has no mobility. Actuator failure can be divided into three main classes. The first is when no redundant actuation is needed as the joint for which the actuator failure occurs will be locked. The second case is when the actuator failure occurs in a set of joint which generates an internal motion of a sub-chain. In this case actuator redundancy must be placed in this set of joints. When the joint does not generate an internal motion and is not locked, actuator failure can be compensated by redundancy in any part of the mechanism which is not locked, including a joint that generates internal motion. In this case the redundant joint guarantees fault tolerance also with respect to the joints that generate the self motion. In general, the chosen redundant actuators must, as a group, generate any self-motion and all the components of the end-effector motion.

\section{Acknowledgement}

The authors wish to acknowledge the support of the Norwegian Research Council and the TAIL IO project for their continued funding and support for this research. The TAIL IO project is an international cooperative research project led by StatoilHydro and an R\&D consortium consisting of ABB, IBM, Aker Solutions and SKF.

\section{References}

Dai, J. S., H., Z., and Lipkin, H. Mobility of overconstrained parallel mechanisms. Transactions of ASME, 2006. 128.

From, P. J. and Gravdahl, J. T. Fault tolerance of parallel manipulators with passive joints. Submitted to SafeProcess, Barcelona, Spain, 2009.

Hervé, J. M. Analyse structurelle des mécanismes par groupe des déplacements. Mechanism Machine Theory, 1978. 13(4).

Lipkin, H. and Duffy, J. Sir Robert stawell Ball and methodologies of modern screw theory. Proc. of Journal of Mechanical Engineering Science, 2002. 216 No 1.

Matone, R. and Roth, B. In-parallel manipulators: A framework on how to model actuation schemes and a study of their effects on singular postures. Trans of ASME, 1999. 121.

Meng, J., Liu, G., and Li, Z. A geometric theory for analysis and synthesis of sub-6 dof parallel manipulators. IEEE Transactions on robotics, 2007. 23, no. 4 . 
Murray, R. M., Li, Z., and Sastry, S. A mathematical introduction to robotic manipulation. CRC Press, 1994.

Rico, J., Gallardo, J., and Ravani, B. Lie algebra and the mobility of kinematic chains. Journal of Robotic Systems, 2003. 20, no. 8.

Rico, J. M., Aguilera, L. D., Gallardo, J., Rodriguez, R., Orozco, H., and Barrera, J. M. A more general mobility criterion for parallel mechanisms. Journal of Mechanical Design, 2006. 128.

Tinós, R., Terra, M. H., and Bergerman, M. A fault tolerance framework for cooperative robotic manipulator. Control Engineering Practice, 2006. 15:615625 . 